\section{Pré-natal na atenção primária do município de João Pessoa-PB: caracterização de serviços e usuárias}

\section{Prenatal primary care in the municipality of João Pessoa, in the Brazilian State of Paraíba: characterization of services and users}

Esther Pereira da Silva 1

Roberto Teixeira de Lima 2

Nadja Laís Soares Ferreira 3

Maria José de Carvalho e Costa 4

\begin{abstract}
Objectives: to characterize prenatal care at Basic Health Units regarding health workers and users in the municipality of João Pessoa, in the Brazilian State of Paraiba.

Methods: a cross-sectional, analytical-descriptive study was carried out. In order to characterize prenatal care, a specific tool was developed based on the guidelines of the Humanization of Prenatal Care and Birth Program, gathering together questions under the headings, structure, process and results.

Results: satisfactory infrastructure was found in the case of most services. In the case of the process, the study identified a low level of adherence to the minimal requirements of the Humanization of Prenatal Care and Childbirth Program (39.92\%), and a low proportion of services including health education (45.5\%) and internal evaluation (47.7\%). In terms of the results of care, a high percentage of pregnant women with clinical irregularities (60.9\%), inadequate nutritional status $(56.3 \%$ overweight or obese) and a low level of exclusive maternal breastfeeding (58.05 \pm 34.19 days) were observed.

Conclusions: aspects of the process relating to prenatal care, the existence of services incorporating health education and internal evaluation indicators and the issue of results involving clinical irregularities, inadequate nutritional status during pregnancy and low levels of exclusive maternal breastfeeding stand in the way of achieving high quality in primary prenatal healthcare.
\end{abstract}

Key words Prenatal care, Primary health care, Quality of health care
1-4 Programa de Pós-graduação em Ciências da Nutrição. Centro de Ciências da Saúde. Universidade Federal da Paraíba. Campus I. $2^{\circ}$ andar. João Pessoa, PB, Brasil. CEP: 58.059-900.

E-mail: robtex@ibest.com.br

\section{Resumo}

Objetivos: caracterizar a assistência pré-natal em Unidades básicas de Saúde envolvendo profissionais e usuárias do município de João Pessoa-PB.

Métodos: estudo do tipo transversal e analíticodescritivo. A fim de caracterizar o pré-natal, um instrumento especifico foi elaborado com base nas diretrizes do Programa de Humanização do Pré-natal e Nascimento reunindo questões distribuídas e analisadas segundo a tríade avaliativa: estrutura, processo e resultados.

Resultados: observou-se boa infra-estrutura em maior parte dos serviços; quanto ao processo, identificou-se baixa adesão aos requisitos mínimos do Programa de Humanização do Pré-natal e Nascimento (39,9\%), além de baixo percentual de serviços com atividades de educação em saúde $(45,5 \%)$ e avaliação interna $(47,7 \%)$. Para os resultados da assistência, altos percentuais de gestantes com intercorrências clínicas (60,9\%), inadequada situação nutricional (56,3\% sobrepeso e obesidade) $e$ baixa prevalência de aleitamento materno exclusivo (58,05 $\pm 34,19$ dias) foram observados.

Conclusões: aspectos de processo referentes à adesão ao pré-natal, presença de serviços com atividades de educação em saúde e indicadores de avaliação interna além de questões de resultados envolvendo intercorrências clínicas, inadequada situação nutricional na gestação e baixa prevalência de aleitamento materno exclusivo são barreiras à qualidade das ações de pré-natal no âmbito da atenção primária à saúde.

Palavras-chave Cuidado pré-natal, Atenção primária à saúde, Qualidade da assistência à saúde 


\section{Introdução}

A assistência pré-natal tem por objetivo reduzir a morbimortalidade materno-fetal. Exames clínicos e laboratoriais oferecidos durante as consultas de prénatal permitem identificar situações de risco e agir precocemente, além da assistência recebida no momento do parto, importante determinante para morbimortalidade durante o período neonatal.1,2

Tem sido constatado que, em nações desenvolvidas, as perdas perinatais estão relacionadas principalmente a doenças de difícil prevenção como: más-formações congênitas, descolamento prematuro de placenta e os acidentes de cordão. Entretanto, em países em desenvolvimento, predominam óbitos perinatais por afecções passíveis de prevenção por meio de adequada assistência pré-natal como síndromes hipertensivas, sífilis congênita e infecção urinária complicada. 3

No contexto da hierarquização dos serviços de saúde pública no Brasil, encontram-se as Unidades Básicas de Saúde (UBS), que constituem a porta de entrada no acesso aos serviços de saúde no âmbito da atenção primária. Devem ser eficientes de tal forma a prevenir, detectar intercorrências clínicas, orientar, acompanhar a gestante durante todo o processo da gestação e no pós-parto além de identificar gestantes de alto risco (na qual a mãe e o feto apresentam riscos de morte) e encaminhá-las a serviços de maior complexidade.2,4

Avaliar o cuidado pré-natal tem grande impacto na redução de resultados obstétricos desfavoráveis, uma vez que a qualidade dessa assistência tem relação estreita com os níveis de saúde de mães e conceptos. 3

A avaliação das práticas de saúde, especificamente a assistência pré-natal, constitui-se em uma potente ferramenta norteadora, para gestores e profissionais de saúde. ${ }^{3} \mathrm{O}$ enfoque mais tradicional de avaliação, proposto por Donabedian, 5 está baseado na teoria dos sistemas, que considera os elementos de estrutura, processo e resultados.

A partir dessa abordagem os profissionais, gestores e políticos podem identificar com mais propriedade fatores que levam a inadequação buscando a melhoria da qualidade das ações em saúde. 6

Em 2000, o Ministério da Saúde instituiu o Programa de Humanização no Pré-Natal e Nascimento (PHPN); até então, não havia um modelo que normatizasse a assistência às gestantes no Brasil. Esse programa estabeleceu não apenas o número de consultas e a idade gestacional de ingresso, mas elencou, também, exames laborato- riais e ações de educação em saúde, e trouxe a discussão das práticas em saúde e suas bases conceituais, em conformidade com os modelos empregados em todo o mundo. ${ }^{7}$

Desde sua implantação até hoje, os dados nacionais referentes à cobertura do Programa de Humanização do Pré-natal e Nascimento apresentam grandes variações por região do país; $;^{7,8}$ considerando-se sete ou mais consultas, observa-se a seguinte distribuição: Norte 30,18\%, Nordeste $39,33 \%$, Sudeste $68,69 \%$, Sul 70,79\%, Centro Oeste $60,38 \%$, e a média no Brasil de 55,38\%.8,9

Inserido na Região Nordeste, encontra-se o município de João Pessoa situado no Estado da Paraíba. No que se refere à atenção ao pré-natal na atenção básica, de acordo com os sistemas de informação do Ministério da Saúde, apresenta uma cobertura de pré-natal de 96,93\%; com $84,81 \%$ das gestantes iniciando o pré-natal no primeiro trimestre; o percentual de mães de nascidos vivos com sete ou mais consultas de pré-natal realizadas está em torno de 59,55\%.10,11 Entretanto, a Paraíba, no contexto nacional, ainda é um dos Estados que apresentaram percentuais altos de nascidos vivos cujas mães não realizaram nenhuma consulta pré-natal. ${ }^{11}$

Diante da importância de verificar a qualidade do cuidado ao pré-natal ofertado nos serviços de saúde, o presente estudo objetivou caracterizar a assistência pré-natal da rede de atenção primária à saúde do município de João Pessoa/PB a partir da tríade avaliativa: estrutura-processo-resultados com base nas diretrizes do Programa de Humanização do Pré-natal e Nascimento.

\section{Métodos}

O presente estudo utilizou dados da dissertação de mestrado: "Proposta de um índice para avaliação do pré-natal de baixo risco".

O delineamento do estudo foi do tipo transversal, de natureza analítico-descritiva, de abordagem quantitativa realizado em unidades básicas de saúde do município de João Pessoa/PB durante o período de Novembro de 2010 a dezembro de 2011. Nesse momento havia 3362 gestantes assistidas em 180 unidades básicas de saúde distribuídas em cinco distritos sanitários de acordo com a localização geográfica de residência.

O cálculo para determinar a amostra das UBS e das usuárias foi segundo o que recomenda Bolfarine e Bussab. 12 para amostragem por conglomerado, considerando a proporção de UBS com atendimento adequado (50\%) para uma significância de 5\% (ou uma confiança de 95\%). A partir deste cálculo, a 
amostra resultou em 44 UBS. Para determinar o número da amostra de usuárias atribuiu-se o percentual de gestantes que fizeram seis ou mais consultas de pré-natal no ano anterior no município, a partir de dados obtidos no Sistema de Informação da Atenção Básica (SIAB), o que totalizou em 254 usuárias (gestantes/puérperas e mães de recémnascidos). A amostra final resultou em 238 usuárias (perda de 6,30\%), pois dezesseis delas apresentaram ausência de informações, ressaltando que pela avaliação do poder do estudo foi suficiente para proporcionar análises consistentes.

Por alocação proporcional, a amostra das UBS foi distribuída entre os cinco distritos sanitários e a das usuárias distribuídas nas UBS selecionadas, excluídas as gestantes de alto risco, as quais o prénatal também era realizado em serviços de maior complexidade.

As variáveis indicativas de estrutura foram os recursos físicos; materiais: equipamentos mínimos para ação; materiais higiênico-sanitários; e medicamentos/suplementos e vacinas importantes para o pré-natal.

Para o processo de trabalho, utilizaram-se as variáveis: de cobertura de mulheres em idade fértil e de gestantes acompanhadas, mínimo de seis consultas de pré-natal, que iniciaram no primeiro trimestre, exames básicos recomendados e imunização antitetânica, multiprofissionalidade nas consultas e realização de procedimentos clínicoobstétricos preconizados no manual do Ministério da Saúde. ${ }^{1}$

A realização de atividades de educação em saúde e uso de indicadores para monitoramento interno do pré-natal também foram aspectos considerados no processo de trabalho.

Para o componente resultados foram consideradas as seguintes variáveis: idade gestacional de início do pré-natal; gestantes em uso de suplementos nutricionais; participação em atividades de educação em saúde; gestantes com no mínimo uma intercorrência clínica durante a gestação; classificação da situação nutricional, ganho de peso gestacional segundo o índice de massa corporal (IMC) pré-gestacional conforme recomendações do Institute of Medicine (IOM), 13 e os desfechos obstétricos.

A coleta dos dados se deu a partir de visitas às unidades básicas de saúde por meio de um instrumento padronizado contendo questões formuladas a partir das recomendações do Programa de Humanização do Pré-natal e Nascimento (PHPN). Os entrevistadores foram treinados previamente e os instrumentos submetidos a pré teste em outras UBS não participantes do estudo. A obtenção das infor- mações ocorreu em três momentos. Primeiro, os dados referentes aos serviços foram coletados a partir de entrevistas aos profissionais de saúde envolvidos diretamente na assistência ao pré-natal (médicos e/ou enfermeiros) e por observação durante as visitas. Segundo, as questões abrangendo as usuárias foram coletadas a partir de entrevistas e registros do cartão das gestantes selecionadas. Neste momento, a data provável do parto era solicitada para um terceiro momento a fim da coleta dos seus dados obstétricos e informações estas obtidas a partir dos registros na caderneta da criança. Especificamente, quanto ao aleitamento materno exclusivo, a informação foi obtida por meio de entrevista às puérperas cujos recém-nascidos tinham idade de no mínimo seis meses completos no momento da visita.

A classificação do pré-natal em categorias de adequação foi feita a partir dos critérios definidos pelos índices de Kessner e do Índice de Adequação da Utilização do Cuidado Pré-natal (APNCU). De modo geral, estes procedimentos utilizam duas informações básicas: a idade gestacional e o número de consultas de pré-natal. O índice de Kessner considera a semana de início do pré-natal, a idade gestacional no parto e o número de visitas de prénatal para classificar o cuidado como adequado, intermediário e inadequado. O cuidado é considerado adequado se o pré-natal é iniciado antes do final do primeiro trimestre de gestação (antes da $13^{\mathrm{a}}$ semana de gestação) e se houver o seguinte número de consultas: uma ou mais consultas para gestação de 13 ou menos semanas; duas ou mais consultas para gestação de 14 a 17 semanas; três ou mais consultas para gestação de 18 a 21 semanas; quatro ou mais consultas para gestação de 22 a 25 semanas; cinco ou mais consultas para gestação de 26 a 29 semanas; seis ou mais consultas para gestação de 30 a 31 semanas; sete ou mais consultas para gestação de 32 a 33 semanas; oito ou mais consultas para gestação de 34 a 35 semanas; e nove ou mais consultas para gestação de 36 ou mais semanas. $\mathrm{O}$ cuidado é considerado inadequado se não houver consultas (ou não forem informadas) em gestação de 14 a 21 semanas; se houver uma consulta ou menos em gestação de 22 a 29 semanas; duas ou menos consultas em gestação de 30 a 31 semanas; três ou menos consultas em gestação de 32 a 33 semanas; e quatro ou menos consultas em gestação de 34 semanas. O cuidado é classificado como intermediário para todas as outras combinações não especificadas acima. ${ }^{14,15}$

O índice APNCU considera a idade gestacional no início do pré-natal e o número de visitas, e classi- 
fica o cuidado pré-natal como inadequado, intermediário, adequado e adequado superior. É considerado como inadequado o início do pré-natal após o quarto mês de gestação (após 16 semanas) ou um número de consultas inferior a $50 \%$ do número recomendado pelo Colégio Americano de Ginecologia e Obstetrícia (ACOG) (13 consultas para gestação de 40 semanas); intermediário se o pré-natal for iniciado até o quarto mês de gestação e forem feitas de 50 a $79 \%$ das consultas recomendadas pelo ACOG; adequado se o pré-natal for iniciado até o $4^{\circ}$ mês de gestação e forem feitas de 80 a $109 \%$ das consultas recomendadas; e adequado superior se o pré-natal for iniciado até o $4^{\circ}$ mês de gestação e forem feitas $110 \%$ ou mais das consultas recomendadas. 14,15

Os dados foram gerenciados e analisados pelos softwares estatísticos: R versão 2.10.1 (Department of Statistics and Mathematics Wirtschafts universitat Wiene) e EPI INFO versão 6.04 (Centers for Disease Control and Prevention, Atlanta, Estados Unidos), a pesquisa seguiu os preceitos éticos referidos na Resolução 196/96 do Conselho Nacional de Saúde do Ministério da Saúde brasileiro, aprovada pelo Comitê de Ética em Pesquisa do Hospital Universitário Lauro Wanderley, da Universidade Federal da Paraíba, sob o protocolo número 660/10. A participação dos profissionais e usuárias no estudo era feito mediante a apresentação, aceitação e assinatura do Termo de Consentimento Livre e Esclarecido.

\section{Resultados}

Quanto aos dados apresentados na Tabela 1, no que se refere à infraestrutura dos serviços, observa-se que a maior parte dos serviços $(67,3 \%)$ foram construídos especificamente para sediar uma unidade básica de saúde, não adaptados de outras construções; Também em sua maioria $(72,7 \%)$ há identificação explícita quanto aos dias e horários de pré-natal para informação dos usuários, e também no momento das visitas para a maioria dos serviços constatou-se a presença dos equipamentos necessários $(98,1 \%)$, materiais higiênico-sanitários $(95,5 \%)$, e medicamentos/suplementos $(95,5 \%)$ essenciais ao pré-natal além da totalidade dos serviços apresentarem materiais de gestão da infor- mação e apoio para exames laboratoriais no serviço ou de referência.

Para as questões envolvendo o processo de trabalho, houve baixa cobertura de mulheres em idade fértil $(14,8 \%)$ e alta cobertura para gestantes $(97,1 \%)$. Maior parte das gestantes $(83,6 \%)$ iniciou o pré-natal no primeiro trimestre com $89,9 \%$ delas apresentando no mínimo seis consultas de pré-natal. Quando associado o número de consultas à realização dos exames básicos, a cobertura reduziu para 67,6\%. Para a imunização antitetânica observou-se alta cobertura $(94,7 \%)$; esta última, quando associada ao início do pré-natal no primeiro trimestre, número de consultas e exames básicos observou-se que o percentual de cobertura diminuiu para $39,9 \%$. Para a maior parte das gestantes $(77,3 \%)$ houve a participação de mais de dois profissionais de saúde durante o pré-natal. Os serviços em sua totalidade realizavam os procedimentos clínico-obstétricos considerados minimamente necessários ao pré-natal; em $45,5 \%$ dos serviços apresentavam atividades extra-consultas de educação em saúde e em 47,7\% das unidades utilizavam no mínimo um indicador para monitorar internamente a qualidade do prénatal.

A Tabela 2 apresenta os resultados da assistência pré-natal: a média de início do pré-natal das usuárias foi de $11,27 \pm 3,37 \mathrm{com}$ mediana de 11 semanas. A maior parte delas $(95,8 \%)$ referiu fazer uso de suplementos; Observou-se baixa prevalência $(10,9 \%)$ de gestantes que participaram de atividades de educação em saúde, e observou-se que a maioria das usuárias $(60,9 \%)$ apresentou, no mínimo, uma intercorrência clínica durante a gestação. Quanto à classificação da situação nutricional segundo o IMC prégestacional, um pouco mais da metade $(56,3 \%)$ foi classificada como sobrepeso e obesidade (Tabela 2).

Ainda na Tabela 2, quanto aos resultados obstétricos, a prevalência de baixo peso ao nascer foi de $11,8 \%$ e a de prematuridade $17,2 \%$. A média de aleitamento materno exclusivo foi de 58,05 $\pm 34,19$ com mediana de 60 dias.

A Tabela 3 mostra que na maior parte dos serviços analisados, quando classificado o pré-natal pelo índice de Kessner (54,6\%) e o APNCU $(63,9 \%)$, verificou-se que a assistência foi considerada intermediária. 
Infra-estrutura e processo de trabalho da atenção primária de assistência ao pré-natal. João Pessoa, PB, Brasil; 2012. 44 Unidades Básicas de Saúde. 238 usuárias.

\begin{tabular}{|c|c|c|}
\hline Aspectos & $\mathbf{N}$ & $\%$ \\
\hline \multicolumn{3}{|l|}{ Infra-estrutura } \\
\hline Unidades básicas de saúde construídas em prédio próprio & 30 & 67,3 \\
\hline $\begin{array}{l}\text { Unidades com identificação explícita quanto aos dias e horários de pré- } \\
\text { natal }\end{array}$ & 32 & 72,7 \\
\hline $\begin{array}{l}\text { Unidades que dispunham de equipamentos necessários para o pré- } \\
\text { natal }\end{array}$ & 43 & 98,1 \\
\hline Serviços que dispunham de materiais higiênico-sanitários & 42 & 95,5 \\
\hline Serviços com recursos materiais de gestão da informação? & 44 & 100,0 \\
\hline $\begin{array}{l}\text { Unidades que no momento da visita tinham os medicamentos / suple- } \\
\text { mentos necessários as gestantes }\end{array}$ & 42 & 95,5 \\
\hline $\begin{array}{l}\text { Unidades que dispunham de apoio laboratorial (no serviço ou de refe- } \\
\text { rência)? }\end{array}$ & 44 & 100,0 \\
\hline \multicolumn{3}{|l|}{ Processo } \\
\hline Percentual de cobertura de mulheres em idade fértil acompanhadasa & 14.285 & 14,8 \\
\hline Percentual de cobertura de gestantes acompanhadasa & 898 & 97,1 \\
\hline Percentual de gestantes que iniciaram pré-natal no $1^{\circ}$ Trimestre & 199 & 83,6 \\
\hline Percentual de gestantes com no mínimo 06 consultas de pré-natal & 214 & 89,9 \\
\hline $\begin{array}{l}\text { Percentual de gestantes que realizaram no mínimo } 06 \text { consultas + exa- } \\
\text { mes básicos (incluindo HIV) }\end{array}$ & 161 & 67,6 \\
\hline Percentual de cobertura da imunização antitetânicab & 225 & 94,7 \\
\hline $\begin{array}{l}\text { Início } 1^{\circ} \text { Trim. + mínimo } 06 \text { cons. + exames básicos (incluindo HIV) + } \\
\text { cobertura antitetância }\end{array}$ & 95 & 39,9 \\
\hline $\begin{array}{l}\text { Percentual de multiprofissionalidade (outro profissional de saúde além } \\
\text { do médico e enfermeiro) durante o pré-natal }\end{array}$ & 184 & 77,3 \\
\hline $\begin{array}{l}\text { Percentual de realização dos procedimentos clínico-obstétricos de ex- } \\
\text { clusividade do pré-natal }\end{array}$ & 238 & 100,0 \\
\hline $\begin{array}{l}\text { Percentual de serviços que possuem no mínimo uma atividade de edu- } \\
\text { cação em saúde de interesse pré-natal }\end{array}$ & 108 & 45,5 \\
\hline $\begin{array}{l}\text { Percentual de serviços com uso de no mínimo um indicador para moni- } \\
\text { torar internamente a qualidade do pré-natal }\end{array}$ & 113 & 47,7 \\
\hline
\end{tabular}

a Cobertura em relação ao total de mulheres em idade fértil (96.525) e gestantes cadastradas (925) dos serviços analisados;

b Duas doses de antitetânica durante o pré-natal ou dose de reforço em mulheres já imunizadas ou nenhuma dose nas mulheres com imunização completa. 
Tabela 2

Resultados da atenção primária de assistência ao pré-natal. João Pessoa, PB, Brasil 2012. 238 usuárias.

\begin{tabular}{|c|c|c|c|}
\hline Assistência & $\mathbf{N}$ & $\%$ & $\bar{X} \pm D P($ Mediana $)$ \\
\hline Usuárias com uso de suplementos na gestação & 228 & 95,8 & \\
\hline Usuárias com atividades extra-consultas & 26 & 10,9 & \\
\hline Usuárias com no mínimo uma intercorrência clínica durante a gestação & 145 & 60,9 & \\
\hline Média de início do pré-natal (em semanas) & - & - & $11,27 \pm 3,37$ (Mediana: 11) \\
\hline \multicolumn{4}{|l|}{$\begin{array}{l}\text { Classificação da situação nutricional e média de ganho de peso gesta- } \\
\text { cional segundo IMC pré-gestacional }\end{array}$} \\
\hline Baixo Peso & 43 & 18,1 & $10,365 \pm 0,86$ (Mediana:10) \\
\hline Eutróficas & 61 & 25,6 & $12,295 \pm 1,45$ (Mediana:12) \\
\hline Sobrepeso & 90 & 37,8 & $12,612 \pm 1,82$ (Mediana:12) \\
\hline Obesidade & 44 & 18,5 & $13,170 \pm 3,07$ (Mediana:13) \\
\hline \multicolumn{4}{|l|}{ Resultados obstétricos } \\
\hline Prevalência de baixo peso ao nascer $(\leq 2500 \mathrm{~g}$ ) & 28 & 11,8 & \\
\hline Prevalência de prematuridade & 41 & 17,2 & \\
\hline $\begin{array}{l}\text { Média (em dias) de aleitamento materno exclusivo em crianças com } \\
\text { no mínimo seis meses completos }\end{array}$ & - & - & $58,05 \pm 34,19($ Mediana $=60)$ \\
\hline
\end{tabular}

IMC= índice de massa corporal.

Tabela 3

Percentual de adequação do pré-natal segundo os índices de Kessner e APNCU.

\begin{tabular}{|c|c|c|}
\hline Classificação & Kessner & APNCU \\
\hline Adequado superior/intensivo & * & 6,7 \\
\hline Adequado & 42,4 & 26,0 \\
\hline Intermediário & 54,6 & 63,9 \\
\hline Inadequado & 2,9 & 3,3 \\
\hline Sem assistência/ desconhecido & 0,0 & 0,0 \\
\hline
\end{tabular}

* Não existe esta categoria de classificação para este índice; APCU= índice de adequação da utilização do cuidado prénatal. 


\section{Discussão}

Estrutura, processo e resultados são áreas que merecem atenção especial quando se pretende avaliar quaisquer práticas de saúde. A melhor estratégia na busca da qualidade das ações de saúde requer a seleção de um conjunto de indicadores representativos destas três abordagens. ${ }^{6}$ Avaliar a partir destes três componentes permite um diagnóstico mais completo da real qualidade da atenção prénatal. 3

Boa estrutura dos serviços de saúde relacionados a adequadas inter-relações pessoais entre profissionais e gestores do sistema, proporciona melhora no processo de atendimento. ${ }^{16}$ Neste aspecto, para todas as variáveis analisadas, a maior parte dos serviços apresentou boa estrutura. Especificamente quanto aos recursos materiais e apoio laboratorial os achados encontrados estão em conformidade com o estudo de Silveira et al.16 e diferem destes pesquisadores quanto aos medicamentos de uso no pré-natal que no presente estudo mostraram-se presentes em quase a totalidade dos serviços.

$O$ processo de trabalho refere-se às ações desenvolvidas nos serviços de saúde, orientada essencialmente para a análise da atuação profissional no cuidado do binômio saúde/doença da população. Avaliar o processo requer critérios que são delimitados, geralmente, pela resolutividade das ações desenvolvidas. ${ }^{3}$ Neste elemento, ressalta-se a baixa cobertura de mulheres em idade fértil indicando a necessidade de captação deste grupo a fim de iniciar precocemente ações de saúde de promoção, prevenção e educação, as preparando para uma boa gravidez e realização do pré-natal de forma satisfatória. Quanto à cobertura de gestantes acompanhadas, início no primeiro trimestre e no mínimo seis consultas realizadas os resultados encontrados encontram-se em conformidade com achados de outros estudos.7,17,18 Para a cobertura de, no mínimo, seis consultas associada à realização dos exames básicos os achados encontrados foram superiores ao estudo de Grangeiro et al. ${ }^{8}$

A baixa cobertura observada quando associada ao início no primeiro trimestre, mínimo de seis consultas, exames básicos e imunização antitetânica demonstra a necessidade dos serviços em enfatizar aspectos qualitativos da atenção ao pré-natal também evidenciado no estudo de Passos e Moura. ${ }^{19}$ Além do que, até o momento atual, não há um modelo padrão de avaliação que investigue aspectos de qualidade e fatores associados à baixa adesão dos profissionais e gestantes aos procedimentos recomendados pelo Programa de Humanização do Pré-natal e
Nascimento dos serviços de pré-natal da rede pública, portanto, estudos locais como a atual pesquisa devem ser estimulados, pois poderão contribuir para direcionar a formulação de estratégias que melhorem a qualidade do pré-natal, traduzindo-se em uma potente ferramenta para a gestão municipal avaliar a assistência pré-natal ofertada à população e o modelo a investir na reorganização da atenção primária à saúde.

A multiprofissionalidade durante o pré-natal foi verificada na maior parte dos serviços, importante para melhora da efetividade da assistência. ${ }^{20} \mathrm{~A}$ alta cobertura de realização dos procedimentos clínicos obstétricos de importância no pré-natal, e a deficiência da maioria dos serviços em planejar atividades de educação em saúde e utilizar indicadores para monitoramento interno é corroborado com os achados de Silveira et al.16 Merece destaque esta última informação uma vez que autores afirmam que o conhecimento e o acompanhamento do impacto das ações em saúde devem ser viabilizados por meio de indicadores adequados, o que implica na existência de registros locais fáceis, confiáveis e contínuos. 3

Os resultados quanto à semana de início do prénatal apresentaram-se inferiores com achados de outro estudo em âmbito internacional21 apontando diferenças no início da atenção entre países desenvolvidos e em desenvolvimento. Entretanto os achados encontrados podem indicar uma boa captação precoce das gestantes pelos serviços, observado pela média e mediana de início do pré-natal ainda no primeiro trimestre gestacional. $\mathrm{O}$ alto percentual de uso de suplementos torna-se importante uma vez que visam o controle de carências de micronutrientes essenciais para a saúde da mãe e feto. 22 Trevisan et al. 18 assim como no presente estudo observaram maior percentual de gestantes que não participaram de atividades de educação em saúde. Neste aspecto ressalta-se que a disponibilidade de horários foi a queixa mais comum para a não participação.

A alta proporção de gestantes com intercorrências clínicas, merece destaque, pois proporciona riscos à saúde materna e fetal favorecendo a ocorrência de desfechos obstétricos indesejáveis. ${ }^{23}$ Quanto à situação nutricional, ressalta-se a maior proporção de gestantes com sobrepeso atentando para o aumento excessivo de peso ao longo dos $\operatorname{anos}^{24}$ e para o ganho de peso gestacional, observouse inadequação quando comparados às faixas de ganho de peso recomendadas pelo IOM, 13 para as categorias baixo peso, sobrepeso e obesidade. É importante mencionar os estudos de Ludwig e 
Currie 25 que apontaram a importância do ganho de peso gestacional adequado como bom preditor de peso ao nascer satisfatório e proteção quanto à riscos à vida adulta, variável indicativa de resultado de prénatal de qualidade.

O percentual de baixo peso ao nascer $(<2500 \mathrm{~g})$ encontrado supera o percentual de $9,18 \%$ encontrado para a Paraíba em 2009.11 Para a prematuridade os achados encontram-se superiores ao de outros pesquisadores em estudo no sul do Brasil apontando para as diferenças regionais. ${ }^{2}$ A mediana de aleitamento materno exclusivo do presente estudo foi superior à encontrada em João Pessoa em 2009 e quando comparada à mediana nacional. 26 É importante mencionar que os valores encontrados para o tempo de aleitamento materno exclusivo no atual estudo são considerados preocupantes, pois provavelmente indicam a introdução precoce de alimentos desnecessários à saúde do bebê. 27

Cabe aqui ressaltar, a escassez de pesquisas publicadas que reúnam no mesmo estudo estrutura, processo de trabalho e resultados da atenção ao prénatal na atenção primária.7,8,16,17,19 Portanto, merece destaque a construção do instrumento que racionalizou esses componentes em um único procedimento onde foi possível estabelecer uma análise mais completa da situação real do pré-natal, contribuindo substancialmente para os sistemas de informação de saúde possibilitando intervenções mais específicas e eficazes.

Quanto à categorização do pré-natal pelos dois índices, devido a utilização de limites diferentes para definir suas categorias de adequação observa-se diferenças quanto a sua classificação. Essas diferenças podem resultar em conclusões diferentes quanto à situação real do cuidado pré-natal levando a intervenções equivocadas. 28,29

O alto percentual de pré-natal "intermediário" quando utilizado os índices de Kessner e o Índice de Adequação da Utilização do Cuidado Pré-natal (APNCU) e a ocorrência de pré-natal "inadequado" para este último pode ser justificado por apresentarem como limites um número elevado de consultas, nove e treze respectivamente, comparado ao recomendado pelo Ministério da Saúde do Brasil

\section{Referências}

1. Brasil. Ministério da Saúde. Pré-natal e puerpério: atenção qualificada e humanizada. Brasília, DF; 2006. (Normas e manuais técnicos).

2. Cesar JA, Mendoza-Sassi RA, Gonzales-Chica DA, Mano PS, Goulart-Filha SM. Características sociodemográficas e de assistência à gestação e ao parto no extremo sul do Brasil. Cad Saúde Pública. 2011; 27: 985-94. de seis consultas. ${ }^{7}$ A presença de pré-natal classificado como "adequado-superior/intensivo" observado pelo APNCU pode representar o aumento do número de consultas e início precoce do pré-natal ao longo dos anos pelas gestantes brasileiras, variáveis que categorizam estes índices. ${ }^{10}$

\section{Considerações finais}

A partir dos achados encontrados por meio da tríade avaliativa: estrutura-processo-resultados, pode-se concluir que os serviços de pré-natal da atenção primária do município em estudo, apresentaram adequada estrutura no que se refere a espaço físico, presença de materiais de higiene, recursos de gestão da informação, equipamentos, medicamentos e apoio laboratorial. Quanto ao processo, para melhor adequação das ações de pré-natal no âmbito da atenção primária é possível afirmar que os esforços devem ser direcionados para ampliar a cobertura de pré-natal no primeiro trimestre, mínimo seis consultas, exames básicos e vacina antitetânica; além de estimular atividades de educação em saúde e indicadores que monitorem internamente a qualidade do pré-natal.

Quanto aos resultados da assistência, conclui-se que ações visando garantir atividades extra-consultas de pré-natal, o tratamento adequado das intercorrências clínicas da gravidez bem como orientação quanto a bons hábitos alimentares, a baixa prevalência de baixo peso ao nascer e prematuridade, além de orientar a prática do aleitamento materno correta desde a gestação devem ser desenvolvidas.

Ressalva-se a importância da pesquisa, uma vez que os resultados observados em discussão juntamente com as equipes de saúde dos serviços analisados, subsidiarão iniciativas para otimizar os recursos materiais e humanos disponíveis, além de garantir aumento na adesão aos procedimentos requisitados pelo Programa de Humanização do Prénatal e Nascimento por parte dos profissionais e gestantes, oferta de cuidado pré-natal de qualidade bem como contribuir para resultados gestacionais e obstétricos favoráveis.
3. Anversa ETR, Bastos GAN, Nunes LN, Pizzol TSD. Qualidade do processo da assistência pré-natal: unidades básicas de saúde e unidades de Estratégia Saúde da Família em município no Sul do Brasil. Cad Saúde Pública. 2012; 28: 789-800. 
4. Martins EF. Mortalidade perinatal e avaliação da assistência ao pré-natal, ao parto e ao recém-nascido em Belo Horizonte, Minas Gerais [tese]. Belo Horizonte: Universidade Federal de Minas Gerais; 2010.

5. Donabedian A. An introduction to quality assurance in health care. New York: Oxford Univ. Press; 2003. 450p.

6. Kobayashi H, Takemura Y, Kanda K. Patient perception of nursing service quality: an applied model of Donabedian's structure-process-outcome approach theory. Scand J Caring Sci. 2011; 25: 419-25.

7. Andreucci CB, Cecatti JG. Evaluation of the Program for Humanization of Prenatal and Childbirth Care in Brazil: a systematic review. Cad Saúde Pública. 2011; 27: 1053-64.

8. Grangeiro GR, Diógenes MAR, Moura ERF. Atenção prénatal no município de Quixadá-CE segundo indicadores de processo do Sisprenatal. Rev Esc Enferm USP. 2008; 42: $105-11$.

9. Brasil. Rede Interagencial de Informações para a Saúde (RIPSA). Indicadores e dados básicos - Brasil - 2008 Cobertura de consultas de pré-natal. Brasília: Ministério da Saúde; 2008. [acesso em 20 ago 2011]. Disponível: http://tabnet.datasus.gov.br/cgi/deftohtm.exe?idb2008/f06. def

10. Brasil. Ministério da Saúde. Secretaria de Vigilância em Saúde. Saúde Brasil 2009: uma análise da situação de saúde e da agenda nacional e internacional de prioridades em saúde. Brasília, DF; 2010.

11. Brasil. Ministério da Saúde. Informações de Saúde. Cadernos do pacto pela saúde 2010/2011 João Pessoa. [acesso em $21 \mathrm{dez}$ 2011]. Disponível em: http://tabnet. datasus.gov.br/cgi/pacto/2010/pb.htm.

12. Bolfarine H, Bussab WO. Elementos de amostragem. São Paulo: Edgard Blucher; 2005.

13. IOM (Institute of Medicine). Nutrition during pregnancy. Washington, DC: Nacional Academy Press; 1990.

14. Alexander GR, Kotelchuck M. Quantifying the adequacy of prenatal care: a comparison of indices. Public Health Rep. 1996; 111: 408-18

15. Kessner DM. Infant death: an analysis of maternal risk and health care. Washington, DC: Institute of Medicine, National Academy of Sciences; 1973.

16. Silveira DS, Santos IS, Costa JSD. Atenção pré-natal na rede básica: uma avaliação da estrutura e do processo. Cad Saúde Pública. 2001; 17: 131-9.

17. Gonçalves R, Urasaki MBM, Merighi MAB, D’Avila CG. Avaliação da efetividade da assistência pré-natal de uma unidade de saúde da família em um município da grande São Paulo. Rev Bras Enferm. 2008; 61: 349-53.
18. Trevisan MR, De Lorenzi DRS, Araújo NM, Ésber K. Perfil da Assistência pré-natal entre usuárias do Sistema Único de Saúde em Caxias do Sul. RBGO. 2002; 24: 293-9.

19. Passos AA, Moura ERF. Indicadores de processo do Programa de Humanização no pré-natal e nascimento no Ceará, Brasil: análise da série histórica 2001-2006. Cad Saúde Pública. 2008; 24: 1572-80.

20. Francischini AC, Moura SDRP, Chinellato M. A importância do trabalho em equipe no programa saúde da família. Investigação. 2008; 8: 25-32.

21. Beeckman K, Louckx F, Putman K. Determinants of the number of antenatal visits in a metropolitan region. BMC Public Health. 2010; 10: 527-36.

22. Long KZ, Garcia C, Ko G, Santos JI, Al Mamun A, Rosado JL, DuPont HL, Nathakumar N. Vitamin A Modifies the Intestinal Chemokine and Cytokine Responses to Norovirus Infection in Mexican Children. J Nutr. 2011; 141: 957-63.

23. Lindstrom E, Hossain MB, Lonnerdal BO, Raqib R, Arifeen SE, Ekstrom EC. Prevalence of anemia and micronutrient deficiencies in early pregnancy in rural Bangladesh, the MINIMat trial. Acta Obst Gynecol Scand. 2011; 90: 47-56.

24. Popkin BM, Doak C. The obesity epidemic is a worldwide phenomenon. Nutr Rev. 1998; 56: 106-14.

25. Ludwig DS, Currie J. The association between pregnancy weight gain and birthweight: a within-family comparison. Lancet. 2010; 32: 60751-9.

26. Brasil. Ministério da Saúde. Secretaria de atenção a saúde Departamento de Ações Programáticas e Estratégicas. II Pesquisa de prevalência de aleitamento materno nas capitais brasileiras e Distrito Federal. Brasília, DF; 2009. (Série C. Projetos, Programas e Relatórios)

27. Vennemann MM, Bajanowski B, Brinkmann G, Jorch K, Yücesan CS, Mitchell EA. Does breastfeeding reduce the risk of Sudden infant death syndrome? Pediatrics. 2009; 123: e406- 11.

28. Suñol R. Promoting research into healthcare accreditation/external evaluation: advancing an ISQua initiative. Int J Qual Health Care. 2009; 21: 27-38.

29. Vanderweele TJ, Lantos JD, Siddique J, Lauderdale DS. A comparison of four prenatal care indices in birth outcome models: comparable results for predicting small-for-gestational-age. J Clin Epidemiol. 2009; 62: 438-45.
Recebido em 2 de fevereiro de 2012

Versão final apresentada em 26 de novembro de 2012

Aprovado em 17 de dezembro de 2012 